\title{
Culturas digitais na educação do Século XXI
}

Simone Lucena*

José Mario Aleluia Oliveira**

\section{Resumo}

As tecnologias digitais estão representadas pela convergência das tecnologias de informática (programas e equipamentos), microeletrônica, telecomunicações, radiodifusão, engenharia genética e optoeletrônica. Essas tecnologias passaram a representar, a partir da década de 70, papel fundamental na economia e no desenvolvimento dos países, gerando, como afirma Castells (1999), uma nova revolução tecnológica. O século XXI está sendo marcado pela universalização das tecnologias digitais e dispositivos móveis conectados em redes, que nos colocam em constante interação com (ciber)espaços sócio-tecnicos onde a comunicação ocorre em lugares não fixos, registrando fatos e informações no instante em que eles acontecem. Os jovens são os que mais utilizam esta forma de comunicação, tornando-a uma marca, um habitus dessa geração caracterizada, dentre outros fatores, pela grande imersão nas culturas digitais. Nesse sentido, o uso cada vez maior dos dispositivos móveis entre os jovens, e sua imersão nas culturas digitais, constituem-se hoje como alguns dos grandes desafios para a educação, principalmente para a formação docentes, pois estes jovens que são autores e produtores nas redes sociais precisam ter outra forma de comportamento, na escola, condizente com um modelo de educação que prioriza a reprodução e a transmissão de informação.

Palavras-chave: Culturas digitais, mobilidade, educação, formação docente

* Doutora em Educação pela Universidade Federal da Bahia. Mestre em Educação pela Universidade Federal de Santa Catarina. E-mail slucen@yahoo.com.br

** Doutor em Educação (2007), na área temática Educação, Conhecimento, linguagem e Arte, pela UNICAMP - Universidade Estadual de Campinas, Mestre em Educação (2001), na área temática Educação, Ciência e Tecnologia, também, pela UNICAMP - Universidade Estadual de Campinas. E-mail:jmaleluia@hotmail.com 


\section{Digital cultures in the 21 st century education}

Abstract

Digital technologies are represented by the convergence of computer technologies (software and hardware), microelectronics, telecommunications, broadcasting, genetic engineering and optoelectronics. Beginning in the 70's, these technologies started playing a keyrole in the economy and development of the countries, generating, as stated by Castells (1999), a new technological revolution. The 21 th century is being affected by the universalization of digital technologies and mobile devices which are connected to networks, putting us in constant interaction with (cyber) socio-technical areas where communication occurs in non-fixed places, recording facts and information the moment they occur. Young people are the ones who use this form of communication the most, making it into a brand, a habitus of this generation that is characterized, among other factors, by a great immersion in digital cultures. In this sense, the growing use of mobile devices among young people as well as their immersion in digital cultures are today some of the major challenges for education, mainly for teacher training, because these young people who are authors and producers of information in social networks, need to change their behavior at school in order to adjust to a model of education that prioritizes the reproduction and transmission of information.

Keywords: Digital cultures, mobility, education, teacher training

\section{Culturas digitales en la educación del siglo XXI}

Resumen:

Las tecnologías digitales están representadas por la convergencia de las tecnologías informáticas (programas y equipos), microelectrónica, telecomunicaciones, radiodifusión, ingeniería genética y optoelectrónica. Esas tecnologías han llegado a representar, desde la década de 1970, papel clave en la economía y en el desarrollo de los países, lo que genera, según lo declarado por Castells (1999), una nueva revolución tecnológica. El siglo XXI se está caracterizando por la universalización de las tecnologías digitales y dispositivos móviles conectados a redes que nos ponen en constante interacción con (ciber)espacios socio-técnicos donde se produce la comunicación en lugares no fijos registrando hechos e informaciones en el momento que ocurren. Los jóvenes son los que más utilizan esta forma de comunicación por lo que es una marca, un habitus de esa generación caracterizada, entre otros factores, por la gran inmersión en culturas digitales. En ese sentido, el uso cada vez mayor de los dispositivos móviles entre los jóvenes y su inmersión en culturas digitales es hoy un gran desafío para la educación y sobre todo para la formación de profesores, pues, estos jóvenes que son autores y productores en las redes sociales, en las escuelas necesitan tener otra forma de comportamiento coherente con un modelo de educación que priorice la reproducción y la transmisión de información.

Palabras clave: culturas digitales, movilidad, educación, formación docente 


\section{Alguns Aspectos das Tecnologias Digitais na Sociedade Atual}

Desde a década final do século passado e início do século XXI pesquisas realizadas sobre o uso das tecnologias digitais por alguns autores com diferentes perspectivas dentre eles: Lévy (1993, 1996, 1999a, 1999b), Castells (1999, 2004), Machado (1993), Silva (2000), Alves (2000), Santaella (2003), Maia e Castro (2006) vem ganhando espaço como instrumento de aperfeiçoamento e intensificação de práticas sociais, políticas, econômicas, educacionais e de ensino e aprendizagem nas sociedades contemporâneas. Para Lévy (1999a, p. 11), "um movimento geral de virtualização afeta hoje não apenas a informação e a comunicação mas também os corpos, o funcionamento econômico, os quadros coletivos da sensibilidade ou o exercício da inteligência". Segundo esse autor a virtualização ou a presença das redes digitais estão em progressão no mundo contemporâneo e desempenham um papel importante no mundo que está se formando nesse novo milênio com o surgimento de um novo pensar, produzir, se relacionar'?

As tecnologias da inteligência, como define Lévy (1993), são elementos que reorganizam, modificam e promovem a construção de novas estruturas cognitivas. As inovações técnicas como o livro, a televisão, o rádio, a informática, bem como a internet, são tecnologias intelectuais que representam esse novo pensar. Além disso, a conexão de empresas de informática, jogos, telecomunicações, imprensa e televisão formam hoje uma grande indústria multimídia. A indústria eletrônica digital compõe a cultura contemporânea e ao refletir a seu respeito não podemos "deixar de constatar que existe uma enorme quantidade de sistemas maquínicos, em particular a mídia eletrônica e a informática, que incidem sobre todas as formas de produção de enunciados, imagens, pensamentos e afetos". (PARENTE, 2003, p. 14).

Neste sentido, hoje, mais que em outros tempos, o desenvolvimento e a riqueza de um país estão relacionados com sua capacidade de gerar, processar e aplicar informação. A informação e o conhecimento sempre foram valiosos para o desenvolvimento das sociedades, assim como a tecnologia determinou em muito a capacidade de produzir bens. Entretanto, hoje, com o advento de um novo paradigma tecnológico, pautado nas tecnologias digitais, como argumentam Castells (1999), lanni (1999) e Lévy (1999a), entre outros, possibilita-se que a própria informação passe a ser produto do processo produtivo.

As tecnologias digitais podem potencializar formas individualizadas de produção e emissão de informações (todos-todos), podendo transformar a lógica da mídia de massa (um-todos), cujos símbolos são a TV, o rádio, a imprensa, o cinema. A internet pode ser considerada um marco na transformação da lógica da comunicação de massa, pois é possível por todos os que nela interagem, produzir e difundir informações em diversos formatos. Ao promover essa dupla ruptura permite agregar ao cenário midiático e intensificar interações, hipertextualidades, conectividades, flexibilidades, produções e socializações de informações em rede, multimídias e a colaboração e a co-autoria entre os envolvidos. Enfim interatividade!

\section{Culturas Digitais e Mobilidade}

Sem dúvida, o desenvolvimento da informática e das telecomunicações, bem como o diálogo entre essas duas áreas, nas últimas décadas, tornou viável a comunicação entre computadores e a expansão da internet. Com um olhar simplista poderíamos definir a internet como uma rede mundial de computadores que interliga outras diferentes redes, tudo isso possibilitado pela ramificação de linhas telefônicas. Entretanto, defini-la apenas pelos seus aparatos técnicos e físicos impossibilita a compreensão das suas principais características.

As tecnologias digitais estão representadas pela convergência das tecnologias de informática (programas e equipamentos), microeletrônica, telecomunicações, radiodifusão, engenharia genética e optoeletrônica. Essas novas tecnologias passaram a representar, a partir da década de 70, papel fundamental na economia e no desenvolvimento dos países, gerando, como afirma Castells (1999) uma nova revolução tecnológica. Esta, segundo o referido autor, "diferentemente de outra revolução, o cerne da transformação que estamos vivendo na revolução atual refere-se às tecnologias da informação, processamento e comunicação" (CASTELLS, 1999, p. 50) 
Portanto, é importante entendermos o contexto da comunicação contemporânea e seus impactos educacionais, sociais, políticos, econômicos, culturais, filosóficos, tanto para os incluídos quanto para os excluídos do processo gradativo de digitalização das sociedades. Também compreender como esses meios digitais podem contribuir significativamente para a ampliação de espaços de cidadania, de participação social, de acesso aos direitos individuais e coletivos, de acesso à informação e ao conhecimento, à leitura e à escrita no mundo contemporâneo, à aprendizagem e ao ensino, enfim, de mais equidades de oportunidades aos brasileiros em geral (Eisenberg e Cepik, 2002).

A digitalização da informação e da comunicação é, sem dúvida, uma das marcas expressivas das sociedades contemporâneas e vêem contribuindo significativamente para mudanças expressivas nas formas de produzir, armazenar e difundir informações. Nesse sentido percebemos que a primeira década do século XXI foi marcada por outra transformação tecnológica causada pela freqüente utilização das tecnologias móveis conectadas em redes. Os tablets, netbooks, smartphones e outros dispositivos móveis tem possibilitado uma comunicação desprendida de lugares fixos e que utiliza diferentes linguagens e novos processo sócio-técnicos próprio deste novo ambiente informacional e da cultura da mobilidade. Esta comunicação móvel materializa o sentido da expressão "tudo ao mesmo tempo agora o tempo todo".

Segundo Castells (1999), nas cidades pós-industriais, o tempo não estrutura mais o espaço e sim o espaço - não físico, o espaço do fluxo de informações - estrutura o tempo. Já para Martin Barbero (1996), as cidades entraram no novo paradigma informacional, centrado no conceito de fluxos, com três fatores principais: a desespacialização, a descentralização e a desurbanização. Há uma transformação espacial ocorrendo nas sociedades contemporâneas. O espaço está virtualizando-se e se transformando em fluxos de informação.

O que estamos denominando aqui de culturas digitais ou cibercultura são as formas de usos e apropriações dos espaços virtuais feitas pelos sujeitos culturais. Para Lévy (1999b, p. 17) a cibercultura "é o conjunto de téc- nicas (materiais e intelectuais), de práticas, de atitudes, de modos de pensamento e de valores que se desenvolvem juntamente com o crescimento do ciberespaço" Nesse sentido, Lemos (2009, p. 139) ressalta que a cibercultura não surge apenas por conta do desenvolvimento das tecnologias, mas "por uma confluência entre uma sociabilidade que emergia na década de 1960 e uma posição contrária a alguns discursos hegemônicos da era moderna, a razão, a ciência, a técnica." Este movimento criou uma cultura alternativa, underground que questionava os valores vigentes das elites e buscava a liberdade de expressão.

Com as culturas digitais, as pessoas produzem, socializam culturas, sonhos, desejos, textos, imagens, sons, e a combinação destes. Vivem, constroem e reconstroem suas vidas e relações em seus cotidianos, participam da vida política, econômica, cultural e participam em instituições públicas e privadas. Influenciam e são influenciados pelas mídias em sinergia entre o mundo presencial e digital em múltiplos tempos e espaços.

As culturas digitais são potencializadas a partir do surgimento da Web 2.0. O desenvolvimento desta nova forma de se conectar em rede inicia-se a partir de 2004 quando a empresa americana O'Reilly Media passou a empregar este termo para se referir a uma segunda geração de softwares e aplicativos da internet que possibilitam maior interação entre as pessoas. A Web 2.0 é marcada não apenas pelos novos programas e interfaces disponíveis na internet, mas principalmente por uma nova forma de pensar e interagir entre os praticantes culturais que estão cada vez mais voltados para produzir e publicar do que para consumir informações. Isso muda tudo!!! Pois os softwares sociais como blogs, Twitter, Flick, Myspace, Facebook, Instagram, entre outros, tem como principal objetivo interligar pessoas de diferentes lugares do planeta com os mais diversos fins.

Consideramos, assim, três fenômenos interligados às redes digitais: Complexo de conteúdos (descentralizada, desnacionalizada); Ambiente de conexão (qualquer sujeito pode torna-se emissor); Sistema de interações (cooperação e intervenção direta no meio). 
Vivemos em uma época de crise das antigas ordens de representação e do saber, em grande perplexidade em relação às formas de produção de subjetividade. Cada sociedade possui seus tipos de máquinas (correlato de expressões sociais e delas se fazerem) e, entre outros motivos, elas não podem ser reduzidas nem a seu caráter instrumental, como extensão dos sentidos do ser humano, nem a sua capacidade manipulatória, como fator condicionante da consciência, mas também como possibilidade geradora de outras constituições do aqui e ago$\mathrm{ra}$, de outras sociabilidades.

É neste sentido que Guatarri $(1992,1996)$ considera a informática, as mídias digitais, a internet e a tecnociência formas hiperdesenvolvidas de subjetividades. Linguagens-potências geradoras de um novo "real", de novas constituições do aqui e agora. O novo significa, aqui, a emergência da imaginação no mundo da razão, das sociedades disciplinares modernas, e conseqüentemente, em um mundo que se pode liberar dos modelos disciplinares da verdade.

O tempo tanto nas ciências, quanto nas artes, nas filosofias, nas culturas, põe em crise a verdade e o mundo: o tempo da verdade (verdades eternas) se substitui a verdade do tempo (múltiplas verdades, ou seja, o novo como processo). Para Bérgson (1990), "ou o tempo é invenção ou ele não é nada".

Atualmente além das culturas digitais vivenciamos a cultura da mobilidade que se desenvolveu com o constante usos das tecnologias móveis conectadas em redes do tipo Wi-fi, WiMax, etc. Estas tecnologias, tais como tablets, smartphones, netbooks e demais dispositivos cabem na palma da mão e podem ser carregadas para qualquer lugar criando redes móveis de pessoas e tecnologias nômades localizadas em diferentes espaços geográficos do planeta. Esta mistura de espaços físicos e ciberespaço cria o que Santaella $(2007,2010)$ chamou de "espaços intersticiais" potencializados pelo uso dos dispositivos móveis conectados em redes. Nesse sentido, a autora nos chama a atenção para o fato de que com as mídias móveis temos uma convergência de lugar, espaço e mobilidade não podendo mais pensá-los separadamente. Ao utilizar o telefone celular uma pessoa passa a ter o dom da ubiquidade, podendo estar em dois lugares ao mesmo tempo, e ambos vão para um segundo plano para favorecer um terceiro lugar, o espaço comunicacional que, nesse caso, coloca as pessoas em uma situação de presença ausente significando que elas estão presentes e, ao mesmo tempo, não estão. (SANTAELLA, 2012, p. 102)

O uso de tecnologias móveis principalmente o telefone celular tem aumentado consideravelmente no mundo. No Brasil, segundo dados da Anatel ${ }^{2}$, em 2008 haviam cerca de 150 milhões de Serviço Móvel Pessoal (SMP). Em 2013 este número aumentou para 271,1 milhões e desse total 103,1 milhões utilizam acessos móveis à internet no SMP.

Dados de outra pesquisa realizada pelo Centro Regional de Estudos para o Desenvolvimento da Sociedade da Informação (Cetic.br) ${ }^{3}$ entre setembro a dezembro de 2013 sobre o uso das Tecnologias da Informação e Comunicação na Educação - TIC Educação apontou que em 2013, 85\% das pessoas com 10 anos de idade ou mais usavam telefone celular. Nas classes D e E esta proporção é de $69 \%$ e na área rural é de $73 \%$. No que se refere as atividades desenvolvidas com este dispositivo a pesquisa revelou que $30 \%$ dos usuários de telefone celular acessam redes sociais; $26 \%$ compartilham fotos, vídeos ou textos; $25 \%$ acessam e-mails; e $23 \%$ baixam aplicativos. O público que mais utiliza esta tecnologia são os mais jovens com faixa etária entre 10 a 15 anos com um percentual de $75 \%$.

O crescimento acelerado do uso dos aparelhos celulares fez com que o desenvolvimento de aplicativos para esta mídia também aumentasse bastante nos últimos tempos possibilitando que cada vez mais as pessoas possam interagir utilizando diferentes linguagens escrita, oral e a hipermídia. Esta outra forma de se expressar, de comunicar e de produzir que mistura e remixa diferentes linguagens é a maneira utilizada pela atual geração nascidos sob égide das tecnologias digitais.

Serres (2013) denomina esta juventude de "Polegarzinha" pelo fato de usarem os dedos polegares para digitais mensagens no celular. Mas este não é o único dife- 
rencial apontado pelo autor para falar dessa geração. $O$ que os diferenciam é que

por celular, tem acesso a todas as pessoas; por GPS, a todos os lugares; pela internet, a todo o saber: circulam, então, por um espaço topológico de aproximações, enquanto nós vivemos em um espaço métrico, referido por distâncias. Não habitam mais o mesmo espaço. (SERRES, 2013, p. 19).

Estas reflexões de Serres nos faz pensar que: se esta geração atual imersa nas tecnologias digitais e hiperconectada tem hoje acesso a toda informação, se comunicam e produzem conteúdos, o que então a escola irá oferecer a estes jovens? O que ensinar? Como ensinar?

Não pretendemos aqui responder estas questões com modelos, exemplos ou fórmulas até porque elas não existem. Nosso intensão é pensar algumas possibilidades para a educação com as culturas digitais e da mobilidade bem como sinalizar para a necessidade de ampliar o desenvolvimento de pesquisas nessa área.

Além disso, é inegável a expansão de espaços e tempos nos quais somos educados e educamos, no sentido mais amplo desse conceito. $\mathrm{O}$ ensino, restrito à educação formal nas unidades de ensino, cada vez mais recebe influência e é permeado por inúmeras informações oriundas dos meios de informação e de comunicação analógicos e digitais.

Neste sentido, a pesquisa do Cetic.br, mencionada anteriormente, apresenta alguns dados importantes para pensarmos sobre a educação no Brasil no século XXI num momento em que a disseminação e uso das tecnologias aumenta de forma significativa cotidianamente criando novas formas culturais principalmente entre os jovens que estão presentes nas escolas e nas universidades. O que se percebe é a cada novo desenvolvimento de um meio de comunicação novas formas culturais são criadas pelos praticantes que se apropriam destes meios e recriam usos próprios. Ao longo da história isso aconteceu desde o desenvolvimento do rádio e da televisão, que potencializou a cultura de massa, até a internet e os dispositivos móveis.

\section{Culturas digitais na educação: a experiência com o PIBID/UFS}

A inserção das tecnologias digitais no espaço escolar brasileiro intensificou-se a partir de 1997 principalmente por conta de políticas públicas que tem priorizado colocar nas escolas "laboratórios de informática", notebooks, tablets e lousas digitais. Desta forma, poderíamos imaginar que o problema de inserção dos equipamentos tecnológicos nas escolas públicas já estaria superado. Contudo, contrariado as expectativas governamentais, o investimento despendido para a comprar dessas tecnologias não tem sortido o efeito esperado na melhoria da qualidade da educação, pois a utilização das tecnologias digitais na educação está para além da aquisição de tecnologias.

Falta, dentre outras coisas, infraestrutura na escola tanto no aspecto físico como também de melhoria da rede elétrica e disponibilidade de conexão a internet. Além disso, é preciso pensar na formação de professores, no currículo e na gestão da escola que precisará desenvolver outras formas de interagir com os alunos frente ao uso das tecnologias digitais.

Para pensar, conceber esta escola que hoje é permeada pelas tecnologias e por jovens, que fora da escola vivenciam outras formas de produzir e consumir conteúdos sendo considerados praticantes culturais (CERTEAU, 2011) atuantes nas mídias e redes sociais, é necessário desenvolvermos pesquisas e práticas educativas que possibilitem a compreensão da sociedade contemporânea, dos sujeitos que nela atuam e dos espaços formais e não formais de educação.

O que fica evidenciado nas politicas públicas de inserção das tecnologias na escola é que esta inserção ocorreu muito mais por uma pressão do mercado de informática para a compra de equipamentos para as escolas do que por uma real necessidade da comunidade escolar. Percebe-se que são incompatíveis os custos empregados na aquisição de equipamentos com o discurso apresentado pelas propagandas sobre estas políticas e os resultados alcançados na educação.

Um dos principais problemas apresentados como fator da não utilização das tecnologias digitais na escola é a 
formação de professores. Isso porque em geral, os programas governamentais resumem a formação continuada de professores em pequenos cursos ou oficinas com carga horária insuficientes para reflexão crítica sobre o uso das tecnologias digitais. O que se percebe é que as políticas públicas educacionais de formação para o uso das tecnologias digitais, na maioria das vezes, são pautadas numa perspectiva de incluir o professor num modelo instrumental preparando-o apenas para utilizar aplicativos operacionais onde não se considera a sua autoria na produção dos materiais pedagógicos.

Nos cursos de formação inicial de professores também se percebe que, em geral, as tecnologias não estão inseridas no currículo. De acordo com a pesquisa desenvolvida por Souza (2013), em algumas IES há na "grade curricular" dos cursos de licenciatura apenas uma disciplina relacionada ao uso das tecnologias digitais na educação, porém muitas vezes esta disciplina não possui carga horária obrigatória. Trata-se de uma disciplina optativa ou complementar.

Nos cursos de licenciatura, durante o período de estágio supervisionado, os alunos não são encorajados a criar atividades que utilizem as tecnologias digitais na sala de aula, mesmo quando o local do estágio possui estas tecnologias. Sendo assim, os professores acabam concluindo a formação inicial sem interagir com as tecnologias digitais no processo pedagógico.

Historicamente a construção do conhecimento no ambiente escolar sempre foi realizada por meio da assimilação de conteúdos e informações que muitas vezes são transmitidas pelos professores aos alunos de maneira descontextualizada do seu cotidiano e da sua cultura. Nesta perspectiva, os sujeitos aprendentes não são autônomos e nem incentivados a perceber a realidade que os cercam de maneira crítica. Esta postura, comportamento que a escola espera dos alunos, é muito diferente da maneira como eles estão aprendendo e interagindo com as culturas digitais.

Pesquisas desenvolvidas por pesquisadores da Universidade Federal de Sergipe (UFS) integrantes do Grupo de Pesquisa em Tecnologias Intelectuais, Mídias e Educação contemporânea (Seminalis - seminalis.net), em escolas públicas das cidades de Itabaiana e Aracaju tem apontado que os alunos das escolas investigadas, apesar de no ambiente escolar não utilizarem a internet, estão realizando produções com diferentes linguagens e difundindo nas redes sociais na internet ou por meio do aplicativo WhatsApp no aparelho celular.

As pesquisas também demostraram que os professores, fora da escola, também estão nas redes sociais, também produzem conteúdos, mas esta forma de produzir e compartilhar não está presente na escola. Os professores das instituições escolares que receberam os tablets do Programa Educação Digital do Ministério da Educação (MEC) em 2013 quando utilizam estes equipamentos em atividades pedagógicas é com a função de bloco de notas, de caderno digital para reproduzir informações. Desta forma, todo o potencial inovador, interativo e colaborativo que as tecnologias digitais possibilitam não é considerado, pois elas acabam sendo utilizadas de forma reducionista e reprodutivista.

Pensar novas possibilidades de produção de conhecimento com as mídias na educação é uma das pesquisas que o Grupo Seminalis tem desenvolvido desde 2014 juntamente com o Programa Institucional de Bolsas de Iniciação à Docência (Pibid) com o apoio da Capes. Nesta pesquisa, estão envolvidos alunos da Pós-graduação da UFS dos cursos de mestrado e doutorado, alunos de iniciação científica e os alunos de iniciação a docência do Pibid além das professoras supervisores que atuam nas escolas da educação básica conveniadas ao Pibid.

De acordo com a Portaria no 096 de 18 de julho de 2013, que apresenta o Regulamento do Pibid no seu Anexo I, este Programa é da Coordenação de Aperfeiçoamento de Pessoal de Nível Superior (Capes) que tem por finalidade fomentar a iniciação à docência, contribuindo para o aperfeiçoamento da formação de docentes em nível superior e para a melhoria da qualidade da educação básica pública brasileira. As bases legais desse Programa são: a Lei $\mathrm{n}^{\circ}$ 9.394/1996, a Lei no 12.796/2013 e o Decreto n 7.219/2010.

Na Universidade Federal de Sergipe o Pibid é desenvolvido em todos os cursos de licenciatura dos Campus 
Universitários localizados em São Cristóvão, Itabaiana e Laranjeira. O curso de Pedagogia está presente em dois campus da UFS em São Cristóvão e Itabaiana e em ambos os campus o Pibid é desenvolvido por eixos temáticos. Um destes eixos - Formação de Professores - desenvolve trabalhos, com os bolsistas de iniciação a docência (ID), nas escolas públicas conveniadas ao programa utilizando as tecnologias e as culturas digitais na educação.

Na fase inicial do Pibid, que começou em março de 2014, foi realizado primeiramente uma formação para o uso das tecnologias na educação envolvendo as alunas de ID e as professoras supervisoras das escolas. Esta formação teve uma carga horária de 180 horas na modalidade semipresencial utilizando ambientes virtuais de aprendizagem (Moodle) e redes sociais (Facebook). Os módulos de estudo da formação tiveram como discussão:

- A Geração net e a formação de professores - reflexões sobre a geração atual que está presente nas escolas e universidades utilizando intensivamente as tecnologias digitais - 40 horas

- Culturas digitais e educação - estudos sobre as diferentes mídias digitais e seu uso na educação tais como: vídeo, podcast, blog, interfaces colaborativas gratuitas. -40 horas

- Produzir e Publicar - conhecer as fases para produção e publicação da rede utilizando diferentes formas e linguagens. 40 horas

- Práticas pedagógicas na cibercultura - construção de projetos pedagógicos utilizando mídias digitais na educação. 60 horas

Como resultados desta formação as alunas de iniciação à docência do Pibid puderam adquirir competências necessárias para o desenvolvimento de trabalhos/oficinas envolvendo o uso de diferentes tecnologias na escola:

- O desenvolvimento do pensamento crítico-reflexivo no que se refere à educação e uso das tecnologias, suas interfaces e perspectivas teórico-metodológicas;

- Ampliação dos conhecimentos e vivências teórico-metodológicas de práticas didático-pedagógicas, ressignificando a sua formação em processo e experiências docentes a partir dos diálogos estabelecidos entre a universidade e a escola;
- Ampliação da articulação interdisciplinar de conhecimentos na sua formação envolvendo conhecimentos educacionais e tecnológicos;

- Atuação didática satisfatória utilizando diferente mídias e linguagens para a produção de conhecimentos;

- Qualificação da formação profissional como Educadores/Educadoras e Pesquisadores/Pesquisadoras e cidadãos atuantes no/com o mundo permeado por tecnologias.

Sabemos que esta é uma área de investigação que envolve as tecnologias na educação é relativamente recente se comparada a outras áreas de pesquisas que foram criadas desde o início da pós-graduação no Brasil. Entretanto, as tecnologias e as culturas digitais já fazem parte do cotidiano dos jovens que estão presentes nas escolas. Ao interagirem com estas tecnologias eles estão estruturando seu pensamento dentro de uma outra lógica que difere da maneira moderna com que a escola ensina. Desta forma, temos um grande desafio que é o de repensar a educação, a escola e a formação de professores.

\section{Algumas considerações para um debate necessário}

Trabalhar com as culturas digitais e com as tecnologias móveis na escola não é apenas usar uma nova metodologia de aprendizagem para transmitir conteúdos enfadonhos, mas pode ser pensar nesse novo indivíduo que é praticante cultural e que pensa, produz saberes e que compartilha opiniões, conteúdos e informações nas redes digitais.

É cada vez mais notório, como já analisado aqui, que as crianças e jovens nascidos a partir do início do século XXI atuam imersas nas tecnologias digitais e hiperconectadas têm atualmente acesso a quase todo tipo de informação, se comunicam e produzem conteúdos dos mais diversificados em múltiplas linguagens. A partir desta constatação, professores, pesquisadores da educação, gestores e supervisores educacionais, formuladores de políticas públicas, entre outros, direta ou indiretamente ligados à processos de ensino formal, não podem se furtar a se questionar o que então as escolas contemporâneas podem ou devem oferecer a estas crianças e aos jovens. 
Desta forma, interessante para o debate na educação contemporânea, é nos perguntarmos como a polegarzinha e o polegarzinho se sentem e se relacionam com a escola que oferecemos e que muito pouco tem a ver com as transformações tecnológicas, sociais, culturais, políticas e, sobretudo, de acesso, produção e socialização de conhecimentos no mundo contemporâneo. Ou seja, a escola e seus processos pedagógicos são de momento histórico que a polegarzinha e o polegarzinho não são mais, foram produzidos para sociedades em que o acesso a informação era difícil e que produzir e socializar conhecimentos eram para poucos.

A polegarzinha e o polegarzinho sabem que o conhecimento dos professores, antes guardados a chaves de laborioso alcance, hoje se encontram disponíveis online, acessível de qualquer local, possível de ser compartilhado e reelaborado pelos polegares dos jovens, disponíveis em sua maioria com não mais equívocos que as antigas enciclopédias e livros didáticos e muitas vezes documentado e explicado em múltiplas linguagens.

No fundo, conscientes ou não, esses jovens ao mostrarem desinteresses pelas escolas transmissoras de conhecimentos, conteudistas, que primam em seus processos pedagógicos pela memorização, padronização e universalização, ao tagarelarem enquanto professores discursam, estão apontando a todos que o saber magistral, tal qual inventado nos últimos séculos, não funciona com esses jovens e com as sociedades que eles estão reinventando. Talvez estejam nos informando que o mundo mudou, que os processos pedagógicos e as escolas precisam mudar radicalmente.

\section{Notas}

* Simone Lucena Doutora em Educação pela Universidade Federal da Bahia. Mestre em Educação pela Universidade Federal de Santa Catarina. Graduada em Pedagogia pela Universidade do Estado da Bahia. Professora do Departamento de Educação da Universidade Federal de Sergipe (UFS) Campus Prof. Alberto Carvalho. Professora permanente do Programa de Pós-graduação em Educação da UFS. Membro do Grupo de Pesquisa em Tecnologias Intelectuais, Mídias e Educação Contemporânea (SEMINALIS/UFS/CNPq - http://seminalis.net/). E-mail slucen@ yahoo.com.br
** José Mario Aleluia Oliveira Doutor em Educação (2007), na área temática Educação, Conhecimento, linguagem e Arte, pela UNICAMP - Universidade Estadual de Campinas, Mestre em Educação (2001), na área temática Educação, Ciência e Tecnologia, também, pela UNICAMP - Universidade Estadual de Campinas, doutorado Sanduíche em Pedagogia Social no Sommersemester 2003 na Universität Siegen na Alemanha no Programa Internacional de Doutoramento INEDD DAAD. Ainda, possui graduação em Licenciatura em Matemática - Faculdades Oswaldo Cruz (2004), graduação em Processamento de Dados pela Faculdade Rui Barbosa (1998). Atualmente professor adjunto na Universidade Federal de Sergipe, no Departamento de Educação, no Centro de Educação e Ciências Humanas $\mathrm{CECH}$, membro do EDaPECI (Grupo de Pesquisa Educação a Distância e Práticas Educativas Comunicacionais e Interculturais) e um dos líderes do SEMINALIS (Grupo de Pesquisa em Tecnologias Intelectuais, Mídias e Educação Contemporânea). Professor Permanente do Programa de Pós-Graduação em Educação PPGED/UFS. E-mail: jmaleluia@hotmail.com

1 Maior compreensão em MARTINS, Francisco Menezes e SILVA, Jurenir Machado da (orgs.). A genealogia do Virtual: comunicação, Cultura e Tecnologias do Imaginário. Porto Alegre: Sulina, 2004 e PELLANDA, Campos Nilze e PELLANDA, Eduardo Campos. Ciberespaço: um hipertexto com Pierre Lévy. Porto Alegre: Artes e Ofícios, 2000.

2 Relatório Anual da Anatel - 2013 disponível em http://www. anatel.gov.br/Portal/verificaDocumentos/documento.asp?nu meroPublicacao $=312603 \&$ pub $=$ original $\&$ filtro $=1 \&$ documento Path=312603.pdf

3 A pesquisa TIC Domicílios 2013 está disponível em http://cetic.br/pesquisa/domicilios/indicadores

\section{Referências}

ALVES, Nilda. Decifrando o pergaminho - o cotidiano das escolas nas lógicas das redes cotidianas. In: OLIVEIRA, Inês Barbosa de; ALVES, Nilda (Org.). Pesquisa no/do cotidiano das escolas: sobre redes de saberes. Rio de Janeiro: DP\&A, 2000.

BARBERO, J.Martín., La Ciudad Virtual. Transformationes de la sensibilidad y nuevos escenarios de comunicaión., in Revista de la Universidad del Valle., n. 14, agosto de 1996, Cali., pp. 26-38.

BERGSON, Henri. Matéria e memória. Martins Fontes, S. Paulo, 1990.

CERTEAU, Michel de. A invenção do cotidiano: artes de fazer. Petropólis-RJ: Editora Vozes, 2010.

CANCLINI, Nestor Garcia. Consumidores e Cidadãos: conflitos multiculturais da globalização. Rio de Janeiro: Editora UFRJ, 1999. 
CASTELLS, Manuel. A sociedade em rede. São Paulo: Paz e Terra, 1999.

CASTELLS, Manuel. A Galáxia Internet: reflexões sobre Internet, negócios e sociedade. Lisboa: Fundação Calouste Gubenkian, 2004.

EISENBERG, José; CEPIK, Marco (Org.). Internet e Política: Teoria e prática da democracia eletrônica. Belo Horizonte: Editora UFMG, 2002.

IANNI, Octavio. A sociedade Global. Rio de Janeiro: Civilização Brasileira, 1999.

GUATTARI, Félix. Caosmose: um novo paradigma estético. Tradução de Ana Lúcia de Oliveira e Lúcia Cláudia Leão. Rio de Janeiro: Ed. 34, 1992.

GUATTARI, Félix. Da Produção de Subjetividade. In: Imagem Máquina: A Era das Tecnologias do Virtual, Org. André Parente, tradução: Suely Rolnik, Ed. 34, São Paulo SP, 1996.

LEMOS, André. Cultura das redes: ciberensaios para o século XXI. Salvador, EDUFBA, 2002.

LEMOS, André. Infraestrutura para a cultura digital. In: SAVAZONI, Rodrigo, COHN, Sérgio (orgs.). Cultura digital.br. Rio de Janeiro: Azougue Editorial, 2009.

LEMOS, André. Espaço, Mídia Locativa e Teoria Ator-Rede. In: Anais do XXI Encontro Anual da Associação Nacional dos Programas de Pós-Graduação em Comunicação - COMPÒS. Universidade Federal de Juiz de Fora, junho de 2012.

LÉVY, Pierre. As tecnologias da inteligência: o futuro do pensamento na era da informática. São Paulo: Editora 34, 1993.

LÉVY, Pierre. O que é virtual?. São Paulo: Editora 34, 1996.

LÉVY, Pierre. A inteligência coletiva: por uma antropologia do ciberespaço. São Paulo: Edições Loyola, 1999a.

LÉVY, Pierre. Cibercultura. São Paulo: Editora 34, 1999b.

LUCENA, Simone. Educação e TV Digital: situação e perspectivas. Maceió: EDUFAL, 2012.

MAIA, Rousiley e CASTRO, Maria Céres Pimenta Spínola. (Orgs.). Mídia, esfera pública e identidades coletivas. Editora UFMG, 2006.

MACHADO, Arlindo. Máquina e imaginário: o desafio das poéticas tecnológicas. São Paulo: EDUSP, 1993.

PRETTO, Nelson De Luca. Uma escola sem/com futuro: educação e multimídia. Campinas: Papirus, 1996.

PRETTO, Nelson De Luca. Linguagens e Tecnologias na Educação. In: X ENDIPE - Encontro Nacional de Didática e Prática de Ensino. Anais do X ENDIPE - Encontro Nacional de Didática e Prática de Ensino. Rio de Janeiro, 2000.
PRETTO, Nelson De Luca; SERPA, Luís Felipe Perret. A educação e a sociedade da informação. In: DIAS, P.; FREITAS, C. V. de. (Ed.). Actas da II Conferência Internacional de Tecnologia de Informação e Comunicação na Educação - Challenges 2001. Braga, PT: Centro de Competências/Universidade do Minho, 2001.

PRETTO, Nelson De L. Redes colaborativas, ética hacker e educação. Revista em Educação, vol. 26, n 3, Belo Horizonte, Dec. 2010. Disponível em <http://www.scielo.br/scielo. php?script=sci_arttext\&pid=S0102-46982010000300015>

SANTAELLA, Lucia. Cultura das mídias. São Paulo: Experimento, 1992.

SANTAELLA, Lucia. Culturas e artes do pós-humano: da cultura das mídias à cibercultura. São Paulo: Paulus, 2003.

SANTAELLA, Lucia. Linguagens líquidas na era da mobilidade. São Paulo: Paulus, 2007.

SANTAELLA, Lucia. A ecologia pluralista da comunicação: a conectividade, mobilidade, ubiquidade. São Paulo: Paulus, 2010.

SERRES, Michel. A Polegarzinha: uma nova forma de viver em harmonia e pensar as instituições, de ser e de saber. Rio de Janeiro: Bertrand Brasil, 2013.

SILVA, Marco. Sala de aula interativa. Rio de Janeiro: Quarter, 2000.

SILVEIRA, Sérgio Amadeu da; CASSINO, João (orgs.). Software livre e inclusão digital. São Paulo: Conrad Editora do Brasil, 2003.

SOUZA, A. de G. Entre a prática e a teoria: a inserção das tecnologias da informação e comunicação (TIC) na formação inicial da Universidade Estadual de Feira de Santana. Aracaju: Programa de Pós-graduação em Educação da Universidade Tiradentes, 2013 (Dissertação de Mestrado).

Data de Recebimento: 20 de setembro de 2014

Data de Aprovação: 30 de outubro de 2014

Data de Publicação: 30 de dezembro de 2014 